\title{
Model-based Optimal Control Method for Cancer Treatment Using Model Predictive Control and Robust Fixed Point Method
}

\author{
Bence Czakó*, Johanna Sápi ${ }^{\dagger}$, Levente Kovács ${ }^{\dagger}$ \\ *John von Neumann Faculty of Informatics, Obuda University, Hungary \\ Email: czako.bence@ stud.uni-obuda.hu \\ ${ }^{\dagger}$ Physiological Controls Research Center, University Research, Innovation and Service Center, Obuda University, Hungary \\ Email: \{sapi.johanna,kovacs.levente\}@ nik.uni-obuda.hu
}

\begin{abstract}
Cancerous diseases are being responsible for the death of many around the globe. Treating these illnesses pose a significant challenge to the medical professionals. While conventional methods, chemotherapy or radiotherapy for example, provide a remedy to the issue their side effects are not negligible. In the past few decades new methods have emerged, which could hinder the strength of the side effects and most remarkably, antiangiogenic therapy can make a notable difference in every day cancer treatment. While the technique has many advantages the cost of treatments are often expensive due to the non-personalized administration medical protocols. In this paper a model based solution is provided which aims to lower the medical expenses during the treatment by creating personalized administration plans with the help of control engineering.
\end{abstract}

Index Terms-Model Predictive Control, Robust Fixed Point Transformation, tumor, MPC, RFPT.

\section{INTRODUCTION}

Cancer related illnesses are one of the leading cause of death in the European Union with 1.359.500 causalities in 2016 according to Malvezzi et al. [1]. Conventional cancer therapy methods such as surgical intervention, chemotherapy, and radiotherapy are used widely in order to treat these diseases. While they are proven to be effective in the majority of cases, many side effect arose during the treatment process. In the past decades a new methodology appeared called Targeted Molecular Therapies (TMT) which targets the key mechanisms of tumor growth [2]. This paper focuses of anti-angiogenic therapy which is one of the targeted methods, and it aims to eradicate the vascular network of the tumor. While this method does not annihilate the tumor cells completely it can reduce its size to a safe level without severely affecting the health of the patient. After the tumor has reached its steady-state, conventional therapies can be applied in order to remove the remaining cancerous cells in a way that it causes significantly less side effect compared to a simple traditional treatment. While at first glance the method seems flawless, one should be noted that anti-angiogenic treatment entails significant medical expenses which can not be covered fiscally

This project has received funding from the European Research Council (ERC) under the European Unions Horizon 2020 research and innovation programme (grant agreement No 679681). by the vast majority of people. However these expenses can be decreased by creating personalized treatment plans which aim to reduce the volume of the tumor in the shortest possible time while considering the cost component of the medication. Hence this paper aims to use model based control techniques in order to create the treatment plans with the use of the famous Hahnfeldt model in conjunction with Model Predictive Control and Robust Fixed Point Transformation based control methods.

\section{THE MODEL OF THE SYSTEM}

In order to create a personalized treatment plan it is necessary to have a mathematical model which describes the change in the volume of the tumor under anti-angiogenic intervention. In this paper the Hahnfeldt model is used which is introduced by Hahnfeldt et al. [3] in 1999 and it describes the tumor growth process considering the significant aspects of the anti-angiogenic therapy. While the model is accurate in the vast majority of the cases, one should be alarmed that since its creation scientist has identified many other alternate vascularization methods which govern the tumor growth, Intussusceptive Microvascular Growth or Vessel CoOption for example [4]. The equations that describe the system are the following [3]:

$$
\begin{aligned}
& V^{\prime}=-\lambda_{1} V \ln \left(\frac{V}{K}\right) \\
& K^{\prime}=-\lambda_{2} K+b V-d K V^{2 / 3}-e K g(t) \\
& g(t)=\int_{0}^{t} c(\tau) \exp (-c l r(t-\tau)) \mathrm{d} \tau
\end{aligned}
$$

The first equation describes the change in the volume of the tumor by the Gomperzian tumor growth model. $V$ denotes the volume of the tumor $\left(\mathrm{mm}^{3}\right), K$ is the volume of the vasculature (endothelial) $\left(\mathrm{mm}^{3}\right)$ and $\lambda_{1}$ is the growth parameter of the tumor $(1 /$ day). The second equation governs the change of the vasculature where $\lambda_{2}$ is responsible for the sudden decreasement in the functional vasculature of the tumor $(1 / d a y), b$ is the angiogenic factor $(1 / d a y), d$ describes the cellular blocking mechanisms of the vasculature $\left(1 / n a p \cdot m m^{2}\right), e$ is the inhibition of the vasculature with the 
medication $(\mathrm{kg} / \mathrm{day} \cdot \mathrm{mg})$, and finally $g(t)$ is the concentration of the administered inhibitor $(\mathrm{mg} / \mathrm{kg})$. The last equation describes the concentration of the inhibitor in the patient by incorporating the concentration of the previously administered medications $(\tau<t)$. Function $c(\tau)$ is the rate of administration of inhibitor concentration at $\tau$ and $c l r$ is the clearance of the medication $(1 /$ day $)$.

The model slightly varies if one considers the systems engineering approach. The first part in the second equation on the right hand side disappears, since it has negligible effects on the overall dynamics and also $g(t)$ will be the input of the system. According to Sápi et al. the modified model which will be used in this paper is [5]:

$$
\begin{aligned}
& \dot{x_{1}}=-\lambda_{1} x_{1} \ln \left(\frac{x_{1}}{x_{2}}\right) \\
& \dot{x_{2}}=b x_{1}-d x_{1}^{2 / 3} x_{2}-e x_{2} g(t) \\
& y=x_{1}
\end{aligned}
$$

where $x_{1}=V, x_{2}=K$ and the output of the system is the volume of the tumor. Simulations showed that the final tumor and vasculature volume without treatment is approximately $1.734 \cdot 10^{4} \mathrm{~mm}^{3}$ which will be used as an initial condition. In order to create linear controllers in the following chapters, the model has to be linearized. The linearization was done around the $g(t)=0$ working point and the state-space matrices for arbitrary $x_{1}$ and $x_{2}$ are:

$$
\begin{aligned}
& A=\left[\begin{array}{cc}
-\lambda_{1} \ln \left(\frac{x_{1}}{x_{2}}\right)-\lambda_{1} & \lambda_{1} \frac{x_{1}}{x_{2}} \\
b-\frac{2}{3} d x_{1}^{-1 / 3} x_{2} & -d x_{1}^{2 / 3}
\end{array}\right] \\
& B=\left[\begin{array}{c}
0 \\
-e x_{2}
\end{array}\right] \\
& C=\left[\begin{array}{ll}
1 & 0
\end{array}\right] \\
& D=\left[\begin{array}{ll}
0 & ]
\end{array}\right.
\end{aligned}
$$

\section{Model Predictive Control DESIGN}

In order to tackle the main issue of the paper it is straightforward to use a cost function which takes monetary expenses and treatment time into consideration. Model Predictive Control (MPC) is capable of ensuring this condition in conjunction with additional constraints. As stated in Camacho et al., MPC consists of three main ingredients namely prediction, optimization, and execution [6]. The algorithm predicts how the states evolve in every time step based on the discrete model of the system. After that, an optimization algorithm computes an optimal control sequence which minimizes a cost function that incorporates the above mentioned criterias. In the last step the first member of this sequence is applied to the real system and the procedure repeats.

In this paper, both nonlinear MPC and its linear counterpart is used to create tumor regulating controllers. To this extent the continuous models - both nonlinear and linear - has to be discretized so that it takes the form $x^{+}=f(x, u)$. A simple linear approximation was applied to the model to keep the algorithm computationally reasonable in exchange of precision. The discrete nonlinear model then holds the following form, where $\Delta t$ is the sampling time which has to be fairly small in order to gain accurate approximations:

$$
\begin{aligned}
& x_{1}^{+}=x_{1}+\Delta t\left[-\lambda_{1} x_{1} \ln \left(x_{1} / x_{2}\right)\right] \\
& x_{2}^{+}=x_{2}+\Delta t\left[b x_{1}-d x_{1}^{2 / 3} x_{2}-e x_{2} g\right]
\end{aligned}
$$

In this representation, variables $x_{1}, x_{2}$ and $g$ now depends on $n \in \mathbb{N}$ instead of $t \in \mathbb{R}$, and $x^{+}$denotes the next value of the variable (the $n+1$ step).

The discrete time matrices can be obtained by the following simple equations according to Chen et al. [7]:

$$
\begin{aligned}
A_{d} & =I+\Delta t A \\
B_{d} & =\Delta t B \\
C_{d} & =C \\
D_{d} & =D
\end{aligned}
$$

With equation (4) a nonlinear controller can be created. To this extent a suitable cost has to be chosen which will be denoted by $\ell(n, x, u)$. According to Grüne et al. the cost has to be selected so that $\ell\left(n, x_{*}, u_{*}\right)=0$ and $\ell(n, x, u)>0, \forall x \in$ $X, \forall u \in U$ where $x_{*}$ and $u_{*}$ are the desired states and input signals, $X=\mathbb{R}^{2}$ is the state space and $U=\mathbb{R}$ is the control space [8]. Based on this, $\ell(n, x, u)$ takes the following form:

$$
\ell(n, x, u)=\zeta\left\|x-x_{r e f}(n)\right\|^{2}+\xi\left\|u-u_{r e f}(n)\right\|^{2}
$$

where $\zeta, \xi \in \mathbb{R}$ are control parameters. As one can see, this cost is designed to incorporate time-varying references as well as constant signals thus the controller can handle custom prescriptions which makes the algorithm more flexible in cases where medical professionals has to specify tumor reduction along some trajectory. Because the model is a single-input single-output (SISO) system and the goal is to control the volume, the cost can be further simplified to:

$$
\ell\left(k, x_{1}, u\right)=\zeta\left(x_{1}-x_{r e f}(k)\right)^{2}+\xi u^{2}
$$

With this time-varying prescription a proper cost function can be introduced which a nonlinear optimization algorithm minimizes. The cost function is given by the following equation [8]:

$$
\min _{u(\cdot) \in \mathbb{U}^{N}\left(x_{0}\right)} J_{N}\left(n, x_{0}, u(\cdot)\right):=\sum_{k=0}^{N-1} \ell\left(n+k, x_{u}\left(k, x_{0}\right), u(k)\right)
$$

In equation (8) $x_{0}$ is the current measurement of the states, $N>2 \in \mathbb{N}^{+}$is the prediction horizon, $x_{u}\left(k, x_{0}\right)$ represents the prediction of the system based on the discrete model (4) and it can be computed iteratively by $x_{u}\left(0, x_{0}\right)=x_{0}$, $x_{u}\left(k+1, x_{0}\right)=f\left(x_{u}\left(k, x_{0}\right), u(k)\right), u(\cdot) \in \mathbb{U}^{N}\left(x_{0}\right)$ denotes control sequence of length $N$ which are the free variables in the optimization, and $\mathbb{U}^{N}\left(x_{0}\right)$ is the constrained control 
sequence space for each measurement. It is important to impose constraints on the optimization process beacuse in certain cases the applied dosage may exceed a critical level thus the health of the patient can be jeopardized. The final step in the algorithm is to apply the first control signal of the calculated sequence.

In the case of linear MPC, equation (5) has to be transformed into an augmented state-space model which leads to a simplified cost function according to Wang et al. [9]:

$$
\begin{aligned}
{\left[\begin{array}{c}
\Delta x_{n+1} \\
y_{n+1}
\end{array}\right] } & =\left[\begin{array}{cc}
A_{d} & o^{T} \\
C_{d} A_{d} & 1
\end{array}\right]\left[\begin{array}{c}
\Delta x_{n} \\
y_{n}
\end{array}\right]+\left[\begin{array}{c}
B_{d} \\
C_{d} B_{d}
\end{array}\right] \Delta u_{n} \\
y_{n} & =\left[\begin{array}{ll}
o & 1
\end{array}\right]\left[\begin{array}{c}
\Delta x_{n} \\
y_{n}
\end{array}\right]
\end{aligned}
$$

In these equations, $\Delta x_{n+1}=x_{n+1}-x_{n}, \Delta u_{n}=u_{n}-u_{n-1}$ are incremental variables, and $o=\left[\begin{array}{ll}0 & 0\end{array}\right]$. Two more vectors has to be introduced in which one contains the predictions, and one carries the free variables of optimization in terms of $\Delta u$, hence

$$
Y=\left[\begin{array}{c}
y_{k+1} \\
y_{k+2} \\
\vdots \\
y_{k+N_{p}}
\end{array}\right], \Delta U=\left[\begin{array}{c}
\Delta u_{k} \\
\Delta u_{k+1} \\
\vdots \\
\Delta u_{k+N_{c}-1}
\end{array}\right]
$$

With these vectors and matrices in hand, the predictions can be computed by the following equation:

$$
Y=F x(k)+\Phi \Delta U
$$

where $x(k)$ is the current measured state of the system,

$$
\begin{gathered}
F=\left[\begin{array}{c}
C A \\
C A^{2} \\
\vdots \\
C A^{N_{p}}
\end{array}\right] \\
\Phi=\left[\begin{array}{cccc}
C B & 0 & \cdots & 0 \\
C A B & C B & \cdots & 0 \\
C A^{2} B & C A B & \cdots & 0 \\
\vdots & & & \\
C A^{N_{p}-1} B & C A^{N_{p}-2} B & \cdots & C A^{N_{p}-N_{c} B}
\end{array}\right]
\end{gathered}
$$

$N_{p}$ is the prediction horizon, and $N_{c}$ is the control horizon with $N_{p}<N_{c}$. One should be aware that in vector (12) and matrix (13) the matrices $A, B$ and $C$ are coming from the augmented state-space model. Upon possessing equation (11), the cost function can be formulated as

$$
J_{N_{c}}(x(k), \Delta U)=\left(R_{s}-Y\right)^{T}\left(R_{s}-Y\right)+\Delta U^{T} R_{w} \Delta U
$$

where $R_{w}$ is a weighting $N_{c}$ by $N_{c}$ identity matrix with some constant multiplier $\xi \in \mathbb{R}, R_{s}$ denotes the reference trajectory with $R_{s}=\left[\begin{array}{llll}r_{k+1} & r_{k+2} & \cdots & r_{k+N_{p}}\end{array}\right]^{T}$ and $r_{k+i}(i \in$ $\left.\mathbb{N}^{+}, 0<i \leq N_{p}\right)$ contains the information about the reference trajectory at time instant $k$. Analytic solution exists for $\Delta U$ if there are no constraints and it is given by the following equation:

$$
\Delta U=\left(\Phi^{T} \Phi+R_{w}\right)^{-1} \Phi^{T}\left(R_{s}-F x(k)\right)
$$

While this result is computationally inexpensive, this is just an Linear Quadratic Regulator with finite horizon which implies that better results can be obtained with the infinite horizon case as pointed out by Mayne et al. [10]. Hence this controller is only used for the sake of comparison and the control vector $\Delta U$ is computed by an optimization algorithm that can deal with additional constraints.

\section{Robust Fixed Point Transformation}

The Robust Fixed Point Transformation (RFPT) based adaptive control is a novel control engineering method introduced by Tar et al. in [11]. While it is a nonlinear method, it has the profound advantage that it is easy to design controllers with it contrary to Lyapunov's 2nd method. The only downside is that the controller does not provide an optimal solution in the traditional sense and it is only capable of tracking time-varying reference signals efficiently. However nonlinear methods are more precise by nature, also the paper deals with time-varying reference signals which justifies the use of RFPT. The underlying concept is the realized-response scheme. If one inverts the system model in a fashion that from the reference trajectory a proper control signal can be obtained which then applied to the plant will control the physical system to the desired regime. While in theory the method works, one should be aware that mathematical models are seldom accurate or complete. This results in a realized response which completely differs from the desired case. The role of the RFPT method is to slightly perturb the desired trajectory in every control cycle iteratively so that the realized response meets with the desired. Detailed description about the method can be found in [11],[12],[13]. The first part of the control cycle is a kinematic prescription which is given by

$$
\begin{aligned}
& e_{\text {int }}(t)=\int_{t_{0}}^{t}\left(q^{n}(\tau)-q(\tau)\right) \mathrm{d} \tau, \\
& \left(\frac{\mathrm{d}}{\mathrm{d} t}+\Lambda\right)^{n+1} e_{\text {int }}=0
\end{aligned}
$$

where $q_{n}$ is the reference trajectory, $q$ is the measured state, $n$ is the order of the system and $\Lambda$ is a control parameter. In the case of the Hahnfeldt model this leads to the following prescription:

$$
\begin{aligned}
& e_{i n t}(t)=\int_{t_{0}}^{t}\left(x_{1}^{n}(\tau)-x_{1}(\tau)\right) \mathrm{d} \tau, \\
& \dot{x}_{1}^{d}=\Lambda^{2} e_{i n t}+2 \Lambda e+\dot{x}_{1}^{n}
\end{aligned}
$$

In equation (17) $x_{1}^{n}$ is the reference volume of the tumor, and $\dot{x}_{1}^{d}$ is the output of the kinematic block. This value is given 
to the deform function, which in this simple SISO case is the following fixed point problem:

$$
\begin{aligned}
& G\left(r \mid r^{d}\right) \stackrel{\text { def }}{=}(r+K)\left[1+B \tanh \left(A\left[f(r)-r^{d}\right]\right)\right]-K \\
& G\left(r_{*}^{d} \mid r^{d}\right)=r_{*}^{d} \text { if } f\left(r_{*}^{d}\right)=r^{d} \\
& G\left(-K \mid r^{d}\right)=-K \text { if } r=-K
\end{aligned}
$$

Constants $A, B, K$ are free control parameters, $f(r)$ is the measured signal, $r^{d}$ is the output of the kinematic block, namely $\dot{x}_{1}^{d}$ and $r_{*}^{d}$ is the solution of the fixed point problem for which the realized response of the system coincides with the desired trajectory. Variable $r$ is responsible for the fixed point iteration so that $r_{n+1}=G\left(r_{n} \mid r^{d}\right)$. In order to establish a proper control signal, the inverse of the model has to be constructed. If one takes equation (2) and rearrange the terms, it leads to

$$
x_{2}=x_{1} \exp \left(-\frac{\dot{x_{1}}}{\lambda x_{1}}\right)^{-1}
$$

Differentiating this expression results in

$$
\dot{x_{2}}=\frac{\dot{x_{1} f}+x_{1} f \frac{\dot{x_{1}} x_{1} \lambda-\lambda \dot{x}_{1}^{2}}{\left(\lambda x_{1}\right)^{2}}}{f^{2}}
$$

where $f$ denotes the exponential term in (19). This can be further simplified to

$$
\dot{x_{2}}=\frac{\lambda \dot{x}_{1} x_{1}+\ddot{x}_{1} x_{1}-\dot{x}_{1}^{2}}{f \lambda x_{1}}
$$

Substituting this into the second equation in (2) in conjunction with equation (19) yields

$$
g(t)=-\frac{\lambda \dot{x}_{1} x_{1}+\ddot{x}_{1} x_{1}-\dot{x}_{1}^{2}+\lambda d x_{1}^{8 / 3}-\lambda b x_{1} f}{\lambda e x_{1}^{2}}
$$

which is the inverse model of the system. Applying the output of the deform function to this inverse leads to a proper control signal $g(t)$ which drives the system along the desired trajectory.

\section{Simulation Results}

Multiple simulations were conducted with the tracking controllers in MATLAB/Simulink. The nonlinear MPC prototype is based on the work of Grüne et al., and the optimization uses the fmincon MATLAB routine, while in the linear case routine quadprog were used [8]. The simulation parameters were chosen according to Sápi et al. thus $\lambda_{1}=0,192, b=$ $5,85, d=0,00873$ and $e=0,66$ [5]. The initial value of the tumor was $x_{0}=1.734 \cdot 10^{4}$ in every case because it can be interpreted as a worst-case scenario so one can assume that for smaller values the results are better. For time-varying reference the following prescription was used:

$$
x_{n}(t)=-\tanh (c t)\left(x_{0}-1\right)+x_{0}
$$

Figure 1. depicts the curves for different values of $c$. For the simulations the value were $c=0.1$ since it provides a middle ground between the duration of treatment and the steepness of the curve (which can lead to higher dosages). The argument for the -1 term is that the system can not be controlled to zero tumor volume, which could lead to issues in the case of RFPT controllers. The length of the simulations were 100 days with fixed 0.1 step size and they were conducted using the ODE3 solver of Simulink.

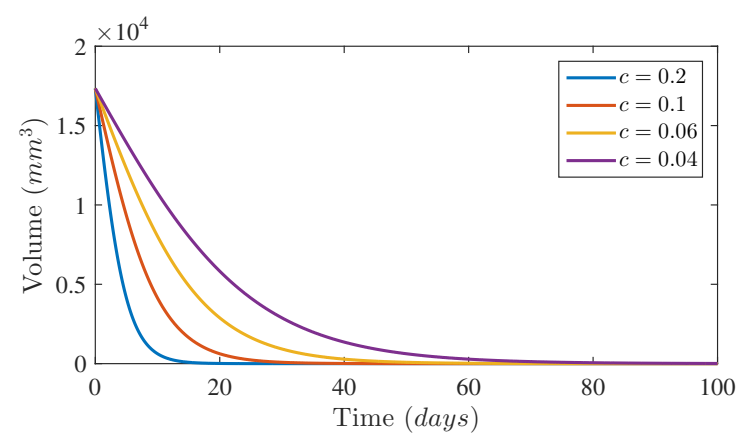

Fig. 1. Reference trajectories for different values of $c$

Multiple values of saturation were imposed on the input signal, namely $25,15,13 \mathrm{mg} / \mathrm{kg}$, which were responsible for keeping the administration at a safe level for the patients. The treatment was successful if the tumor reached $10 \mathrm{~mm}^{3}$ volume or less. The scrutinized attributes of the simulations were the following: the volume of the tumor in steady state $\left(\mathrm{mm}^{3}\right)$, total inhibitor concentration during the treatment $(\mathrm{mg} / \mathrm{kg})$, duration of tumor reduction to $10 \mathrm{~mm}^{3}$ (days), total inhibitor concentration to $10 \mathrm{~mm}^{3}$ volume and steady state inhibitor concentration $\mathrm{mg} / \mathrm{kg}$.

In the case of linear MPC, simulations showed that there is a combination of parameters that provides an optimal solution that can not be improved furthermore regard to the total concentration and duration of the treatment, thus $N_{p}=N_{c}=10, x_{1}=x_{2}=10$ (which are the working points of linearization), and $\xi=1.3 \cdot 10^{4}$ (which corresponds to matrix $R_{w}$ ). The best results were achieved for constant 0 reference volume with $25 \mathrm{mg} / \mathrm{kg}$ saturation for which the reduction time was 44.64 days and the concentration during reduction was $870.7 \mathrm{mg} / \mathrm{kg}$. However the time-varying reference tracking capabilities of the controller were weak which can be attributed to the linear nature of the controller.

For the simulation of the nonlinear MPC, the prediction and control horizon was $N_{c}=N_{p}=10$ because it provided trustworthy results while it was not very expensive computationally. The simulations examined the behaviour of the controller for different levels of saturations, and values of $\zeta$ which were chosen from the interval $\zeta \in[750 ; 1250]$ because for other values the system became unstable as a result of the singularities in the system model. However the weighting factor on the control was $\xi=1$ in every case because it also resulted in pathological behaviour of the system. For constant $x_{1}=0$ reference, the best results came from $\zeta=1000$ for 
$25 \mathrm{mg} / \mathrm{kg}$ saturation. Results for different values of $\zeta$ can be seen in Table I. For time-varying reference, the tracking error remained significant however it was better than the linear case. The tumor reduction can be seen on Figure 2. in conjunction with the treatment protocol on Figure 3.

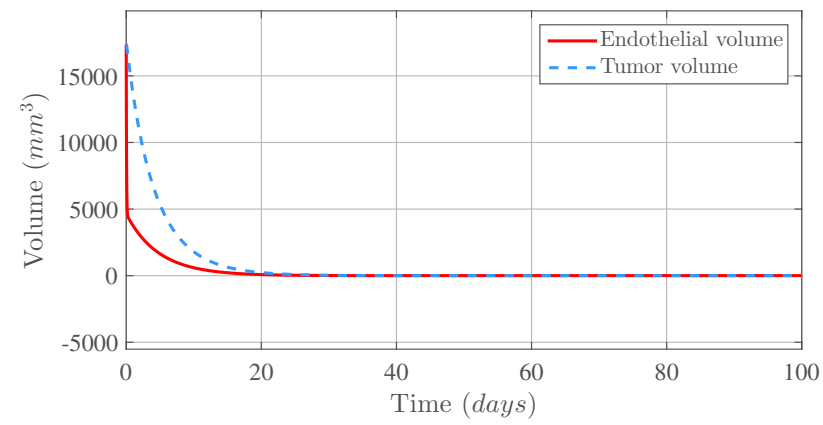

Fig. 2. Tumor reduction with the nonlinear MPC controller

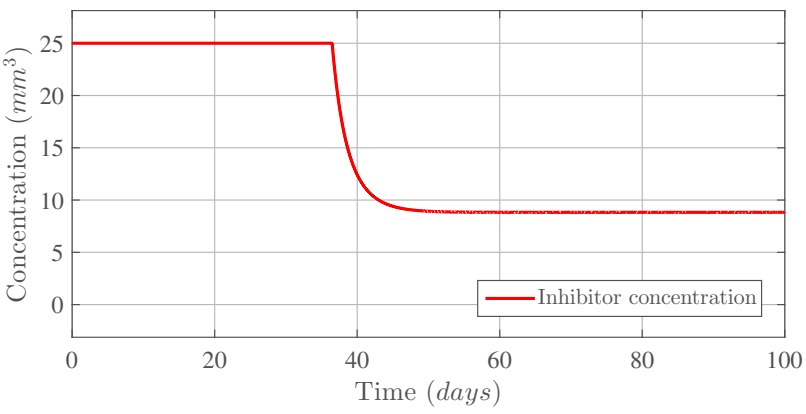

Fig. 3. Administration protocol with the nonlinear MPC controller

The last simulations were performed on the RFPT controller. Since it is only able to track time-varying references, the constant cases were omitted. The controller parameters were $B=-1, K=5,3 \cdot 10^{10}, A=10^{-11}$ and $\Lambda=1$. For the examined reference volume, only saturation $25 \mathrm{mg} / \mathrm{kg}$ was applicable because in other cases, the controller failed to keep the system in the convergence region due to the insufficient maximum amount of admissible inhibitor which is required by controller to maintain a precise tracking. Despite these facts, the RFPT controller vastly outperformed the other MPC variants for tracking time-varying references which means that this method is the best for a flexible treatment. The reduction can be seen on Figure 4. with the tracking error on Figure 5. The produced protocol can be viewed on Figure 6 .

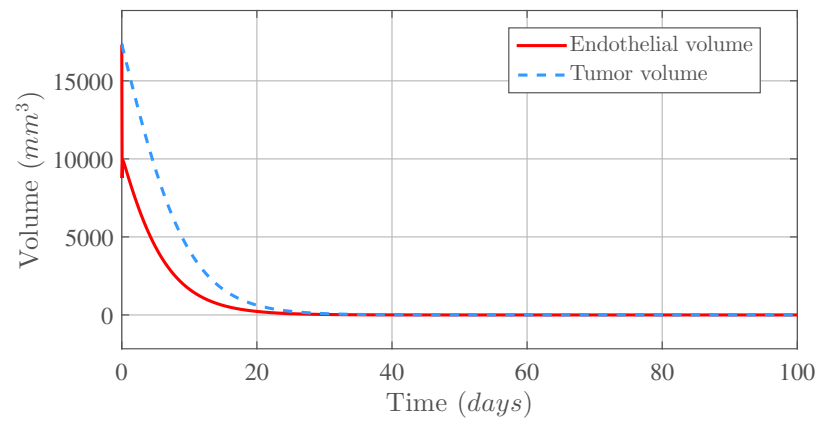

Fig. 4. Tumor reduction with the RFPT controller

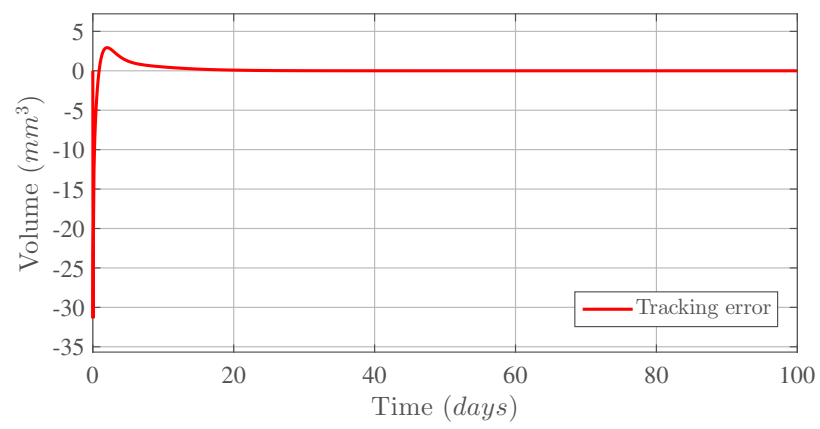

Fig. 5. Tracking error with the RFPT controller

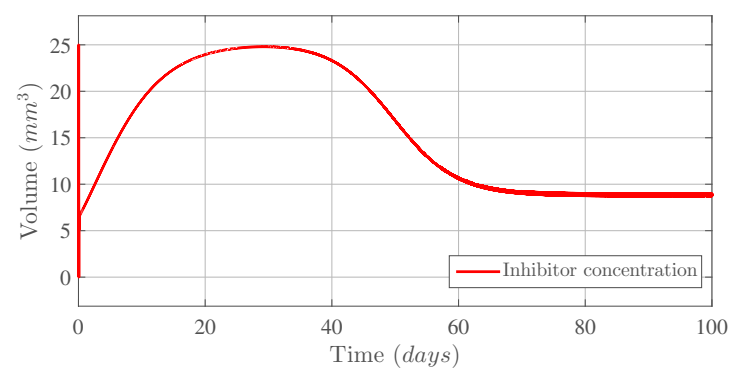

Fig. 6. Administration protocol with the RFPT controller

\section{CONCLUSION}

In this paper, several controllers were designed and tested in order to regulate the volume of a tumor by the help of anti-angiogenic therapy. One can draw the conclusion that linear techniques are not sufficient for solving such an unstable nonlinear problem thus their use in a systems biology environment is highly questionable. However, nonlinear controllers provided excellent results for tracking both constant and time varying reference signals. For the constant case, nonlinear MPC proved to be efficient for different saturation levels, and for time-varying signals RFPT provided accurate results. However it should be noted that the model and the controllers have to be improved. Further work has to be devoted into incorporating new scientific results into the model. For the controllers, one must examine their behaviour under parameter 


\begin{tabular}{|c|c|c|c|c|}
\hline$\zeta$ & $\begin{array}{c}\text { Saturation } \\
(m g / k g)\end{array}$ & $\begin{array}{l}\text { Steady } \\
\text { state } \\
\text { tumor } \\
\text { volume } \\
\left(\mathrm{mm}^{3}\right)\end{array}$ & $\begin{array}{c}\text { Duration } \\
\text { of } \\
\text { reduction } \\
(\text { days })\end{array}$ & $\begin{array}{c}\text { Total } \\
\text { concentration } \\
\text { during } \\
\text { reduction } \\
(m g / k g)\end{array}$ \\
\hline \multirow{3}{*}{750} & 13 & 3,09 & 83,8 & 1089 \\
\hline & 15 & 0,89 & 64,69 & 970,1 \\
\hline & 25 & 0,81 & 36,1 & 891,6 \\
\hline \multirow{3}{*}{850} & 13 & 3,09 & 83,8 & 1089 \\
\hline & 15 & 0,83 & 59,48 & 892,1 \\
\hline & 25 & 0,76 & 36,1 & 890,5 \\
\hline \multirow{3}{*}{1000} & 13 & 3,09 & 83,8 & 1089 \\
\hline & 15 & 0,77 & 59,48 & 892,1 \\
\hline & 25 & 0,7 & 30,6 & 764 \\
\hline \multirow{3}{*}{1150} & 13 & 3,09 & 83,8 & 1089 \\
\hline & 15 & 0,72 & 64,68 & 970,1 \\
\hline & 25 & 0,65 & 36,1 & 894,4 \\
\hline \multirow{3}{*}{1250} & 13 & 3,093 & 78,6 & 1022 \\
\hline & 15 & 0,69 & 59,48 & 892,1 \\
\hline & 25 & 0,628 & 33,3 & 765,8 \\
\hline
\end{tabular}

NONLINEAR MPC PERFORMANCE FOR DIFFERENT VALUES OF $\zeta$

uncertainties and also their stability, but other modern control engineering techniques can be investigated (LPV control [14], fractional-order control [15]). In this paper the preliminary results justify the further researches which can provide a remedy in a safe way for the expenses in anti-angiogenic cancer treatment.

\section{REFERENCES}

[1] M. Malvezzi, G. Carioli, P. Bertuccio, T. Rosso, P. Boffetta, F. Levi, C. L. Vecchia, and E. Negri, "European cancer mortality predictions for the year 2016 with focus on leukaemias," Ann Oncol, vol. 27, no. 4, pp. 725-731, 2016.

[2] P. Charlton and J. Spicer, "Targeted therapy in cancer," Medicine, vol. 44, no. 1, pp. 34-38, 2016.

[3] P. Hanhfeldt, D. Panigrahy, J. Folkman, and L. Hlatky, "Tumor development under angiogenic signaling: a dynamical theory of tumor growth, treatment response, and postvascular dormancy," Cancer Res., vol. 59, no. 19, pp. 4770-4775, 1999.

[4] B. Dome, M. Hendrix, S. Paku, J. Tovari, and J. Timar, "Alternative vascularization mechanisms in cancer," Am J Pathol, vol. 170, no. 1, pp. 1-15, 2007.

[5] J. Sapi, D. Drexler, and L. Kovacs, "Parameter optimization of $\mathrm{h}$ infinity controller designed for tumor growth in the light of physiological aspects," in CINTI201314th IEEE International Symposium on Computational Intelligence and Informatics, 2013.

[6] E. Camacho and C. Bordons, Model Predictive control. Springer London, 2007.

[7] C. Chen, Linear System Theory and Design, 3rd ed. Oxford University Press, Inc., 1998.

[8] L. Grune and J. Pannek, Nonlinear Model Predictive Control. Springer London, 2011.
[9] L. Wang, Model Predictive Control System Design and Implementation Using MATLAB. Springer London, 2009.

[10] D. Mayne, J. Rawlings, C. Rao, and P. Scokaert, "Constrained model predictive control: Stability and optimality," Automatica, vol. 36, no. 6, pp. 789-814, 2000.

[11] J.K.Tar, J. Bito, L. Nadai, and J. T. Machado, "Robust Fixed Point Transformations in adaptive control using local basin of attraction," Acta Polytechnica Hungarica, vol. 6, no. 1, pp. 21-37, 2009.

[12] L. Nadai, I. Rudas, and J. Tar, System and Control Theory with Especial Emphasis on Nonlinear Systems. Typotex, 2012.

[13] B. Czako and K. Kosi, "Novel method for quadcopter controlling using nonlinear adaptive control based on robust fixed point transformation phenomena," in SAMI2017- 15th IEEE International Symposium on Applied Machine Intelligence and Informatics, 2017.

[14] L. Kovacs, "Linear parameter varying (LPV) based robust control of type-I diabetes driven for real patient data," Knowl-Based Syst, vol. 122, pp. 199-213, 2017.

[15] D. Copot, R. De Keyser, J. Juchem, and C. Ionescu, "Fractional Order Impedance Model to Estimate Glucose Concentration: in Vitro Analysis ," ACTA Pol Hung, vol. 14, no. 1, pp. 207-220, 2017. 